\title{
On Representing the Temporal Structure of a Natural Language Text*
}

\author{
Kurt Eberle \\ Institut für Maschinelle Sprachverarbeitung \\ Universität Stuttgart \\ Azenbergstraße 12 \\ 7000 Stuttgart \\ Germany \\ Net address: kurt@adler.philosophie.uni-stuttgart.de
}

\begin{abstract}
A proposal to deal with tenses in the framework of Discourse Representation Theory is presented, as it lias been implemented for a fragment at the IMS for the project LILOG. It is based on the theory of tenses of H. Kamp and Ch. Rohrer. The system uses the tense and aspect information, the information about the temporal discourse structure of the preceding text stored in a specific list of possible reference times, and background knowledge. These types of information interact in order to choose a suited temporal anchor for the event of a new sentence.

Witl respect to extended texts, choosing the right reference time for a new event is a problem which has been largely neglected in the literature.
\end{abstract}

\section{Introduction}

In Reichenbach (1947) the tenses are analyzed into different orderings of the relevant temporal paranseters which are: the time of the event talked about, the speech time (time of utterance) and the reference time. Simple past, for instance, identifies event- and reference-time, which are both before the speech time. In the case of past perfect the event time precedes the reference time which precedes the speech time.

Kamp and Rohrer $(1983,1985)$ were led by textphenomena to split up the Reichenbachian reference time into two contextually given coordinates: the refenence time (in the narrow sense) and the temporal perspective time.

(1) Harry handed the book about giraffes to Jane $\left(e_{1}\right)$. Two hour's ago he had descovered it in Alfred's bookshop (e. $)_{2}$. He had bought it $\left(e_{3}\right)$, had inserted a dedication $\left(e_{4}\right)$ and had it urapped up $\left(e_{5}\right)$. Jane took the book with a smile $\left(e_{6}\right)$

In (1), the events $e_{2}-e_{5}$ are ordered consecutively,

"This research has been done in the context of LILOG. Thanks on this way to the members of the LILOG-research group of IBM for helpfull comments and especially for having made the integration of the suggested system in the LILOGprototype possible. each taking its predecessor as reference time, with the exception that the context does not jrovide an event which precedes $e_{2}$ and which, therefore, could play the role of reference time with respect to $e_{2}$. The whole sequence $e_{2}-e_{5}$ is seen from a vantage point in the past, $e_{1}$, which, therefore, plays the role of perspective tine with regard to the structural integration of $e_{2}-e_{5}$. The perspective from which $e_{1}$ and $e_{0}$ are seen is the speech time. $e_{1}$ serves as reference time for $e_{a}$. In the approach of Kamp and Rohrer the role that the reference time plays in Reichenbach's system is taken over by the perspective time with respect to the meaning of the tenses in single sentence discourses: The meaning (one of the meanings) of past perfect is that the event time precedes the perspective time which precedes the specch time. The meaning of simple past is that the event time precedes the speech time which coincides with the perspective time. However, in addition to a system like Reichenbach's there is another parameter which comes exclusively from the text, the reference time proper, which is part of the impact of the tenses on the temporal reconstruction of a narrative, thus completing the sentential meaning of the tenses by a textual aspect. Here, the idea, that we take over from Kamp and Rohrer, is that in a sequence of simple past sentences introducing events (not states) normally the event of a new sentence follows the reference time which is provided by the event of the preceding sentence within the sequence. By transposition the same should hold for sequences of past perfect sentences. Here, the perspective is shifted from the speech time to a (contextually given) point in the past. There is all additional feature however with respect to temporal parameters. Adverbials can provide a location time for events and states. For instance, in (1), the 10 cation time of $e_{2}$ is the time pointed to by two hours ago.

On the basis of the Reichenbachian approach using three temporal parameters the problem of integrating a new event in the event structure of the preceding text has been attacked by Hinrichs (1986), Partee(1984) and others. More independant of this approach are suggestions made by Moens and Steedman (1988) and by Webber(1988). 
Since we think that a correct analysis of narratives cannot do without a fine grained distinction between the roles played by temporal anchors, our approach uses the coordinates suggested by Kamp and Rohrer. Sucl coordinates are stored in a structured list, called T-list, which reflects the discourse structure of the preceding text. The problem of choosing the right auchor for the new event from the preceding text is largely neglected in the literature. Very often there, the preceding text consists of only one sentence or the problem is restricted to the intra-sentential one presented by temporal conjunctions. How do we decirle in (1) that the reference time for $e_{3}$ is not the earlier introduced $e_{1}$, but $e_{2}$ and that the reference time for $e_{0}$ is not the most recent reference time $c_{5}$, but $e_{1}$ ? In our system we use the structure of the T-list among other things to get a hierachy of the topicality of the reference times. This is very close to the iclea of accessibility in Discourse Representation Theory (Kamp (1981)) and to the notion of focus in the work of Gros' and Sidner (1985). In order to tackle the task of event-integration, which we call "temporal resolution" - making allusion to the very similar problem of the NP-resolution, where anaploric links for the case of nominals have to be established - we structure the T-list along the lines of different discourse relations such as continuation, elaboration, flashback etc. Flasblack, for instance, holds in the example above between $e_{1}$ and $e_{2}$ and between $e_{1}$ and $e_{3}$. The relation between $e_{2}$ and $e_{3}$ is continuation. As we will see in the section after next, often, from the purely linguistic point of view there are alternatives with respect to the decision about the discourse relation which directs the integration of a new event or state.

In our system, the criteria to decide about the relevant discourse relation are provided by the tense forms of the reference event(s) and of the new event respectively, by the existence and the characteristics of temporal adverbials, by the aspect of the events - is it an event proper or a state - and by internal semantic properties of the events which are made explicit by means of rules stored in the background knowledge. Of course, in addition, the hierarchically structured T-list influences the cloice and helps to update the system correctly, where updating means adding a new temporal condition to the representation of the text and anding a new reference time to the T-list at a specific place.

In the next section we will briefly introduce the kind of discourse representation structures that we use. 'Then we will say something about the discourse rclations dealt with and we present the syntax of the T-list. After that we sketch the relevant background knowledge and finally, we give some of the resolution rules of the system.

The structuring of the T-list extents a suggestion of Elserle and Kasper (1989) and comprises elenents of a jroposal by Reyle (1986).

The algorithm suggested is part of the LILOGprototype which is used for text understanding. In particular it nses knowledge provided by the knowledge base and the inference component of the prototype.

\section{DRT and Events}

Within the framework of Discourse Representation Theory (DR') (Kantp(1981)) a Discourse Representation Structure (DRS) is a pair $\langle U, K\rangle$ consisting of a set $U$ of discourse referents (DRFs) and a set $K$ of conditions. DRSs are interpreted modeltheoretically. DRT uses a variant of the Davidsonian method of talking about events: they are treated as a kind of objects.

In LILOG we use DISs for text representations. But we deviate from the classical DRT-style in using one-phice event predicates and thematic roles instead of n-place event predicates. A second difference is given by the fact that in LILOG DRSs come with an index. The index is the list of reference times available with respect to the DRS in question. For a simple sentence it will be a list consisting only of one element - the DRF of the event introduced - for a text it will be a complex list, the T-list.

So, for instance, the LILOG-DRS for coming of $x$ can be illustrated as follows:

$$
<[e],\left[\begin{array}{l}
e, x \\
\text { come }(e) \\
\text { agent }(e)=x
\end{array}\right]
$$

For the DIRS to be valid there must exist an embedding function which maps $c$ onto an event of the model structure sucl that $e$ satisfies the conditions as described in the IJRS.

\section{Discourse Relations}

It is clear that the idea about the order of events introduced by sequences of sentences that we have sketched in the introduction is an oversimplification.

In the following we will restrict ourselves to the tenses prevailing in narratives, simple past and past perfect, and to only some of the text phenomena related to these tenses (as we have done in the LILOG implementition with respect to the German connterparts of these tenses).

Besides the discourse relations used in exanple (1), i.e. flashback and continuation, there are at least two more. which we call elaboratzon and background.

Compare the two following examples:

(2) Monday Hans went to Paris. Tuesday he met Gali.

(3) Monday Haths went to Paris. At the border he had some trouble.

(2) describes a continuation. This can be deduced from the characteristics of the location times. 
In contrast to that, (3) describes an elaboration. We call the relation between a new event $e_{2}$ and its reference event $e_{1}$ an elaboration, if $e_{2}$ describes $e_{1}$ on a more fine-grained level (which gives rise to the temporal condition of inclusion ( $\subseteq$ ) between the ncw event and the reference event within the representation of the text).

In (3) the relation of elaboration can be deduced only if there is some input from the knowledge base equipping the resolution component with facts about journeys to foreign countries. Notice that, here, the temporal inclusion is due to the homomorphic local inclusion of the border into the path of the travel. This illustrates that an intelligent temporal resolution component cannot do without having access to local reasoning.

Now compare the following pair of examples, taken from Dowty (1986) and from Hinrichs (1986) respectively:

(4) Joht entered the president's office. The clock on the wall ticked loudly.

(5) Jameson entered the room, shut the door carefully and switched off the light. It was pitch-dark around him because the venetian blinds were closed.

In both (4) and (5) the last sentence introduces not an event proper, but a state. As widely accepted, the normal case of relating a new state $e_{2}$ to an existing reference event $e_{1}$ seems to be that of inclusion $\left(e_{1} \subseteq e_{2}\right){ }^{1}$

However, as examples like (5) make clear, this default can be overwritten. Here too, it seens that background knowledge gives the decisive indication about when the default has to be upset.

We call the discourse relation of (4) background and that of (5) causally introduced background. For cases like (5) we introduce the new state $e_{2}$ as following the actual reference event $e_{1}\left(e_{1} \prec e_{2}\right)$ and as including a dummy reference time $t\left(t \subseteq e_{2}\right)$ with respect to which $e_{2}$ introduces a background. In the further course of processing the text, $t$ has to be instantiated by a new event which is understood as following $e_{1}$.

The T-list makes use of these discourse relations as structuring elements. For instance, processing the sample text (6), which integrates the text phenomena reflected by the examples (1) - (4), should result in a representation which has as index the T-list (7):

(6) John left Paris by the Porte de $S^{t}$ Cloud late in the evening $\left(e_{1}\right)$. He went to Frankfort $\left(e_{2}\right)$.

At the border he was stopped $\left(e_{3}\right)$. It was pretty dark $\left(e_{4}\right)$. Only a few lights were burning $\left(e_{5}\right)$. He had to open hat boot $\left(e_{6}\right)$. In Paris he had bought five cartons of rigarettes $\left(e_{7}\right)$ and had hidden them in the car $\left(e_{8}\right)$. The customs officers missed them (eg).

\footnotetext{
'This parallels, for instance, the insights of Partee(1984), Hinrichs(1986), Kamp and Rohrer(1985) and others.
}

Early in the morning John entered a bar near the mess tower in Frankfort $\left(e_{10}\right)$, ordered a beer $\left(e_{11}\right)$ and lighted a cigarette $\left(e_{12}\right)$. He was happy $\left(e_{13}\right)$.

(7)

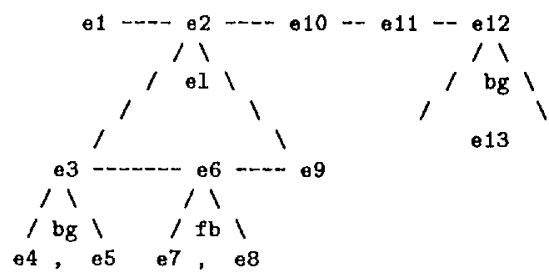

In (7) the structure of (6) is stored in the following way: $e_{1}, e_{2}, e_{10}, e_{11}, e_{12}$ (the leaving of Paris, the going to Frankfort, the entering the bar, the ordering of beer and the lighting the cigarette) on the one hand and $e_{3}, e_{B}, e_{9}$ (the stopping, the opening of the boot, the failure of the officers) on the other form continuation chains (tied up in the picture by - ) . The second of these chains elaborates an event of the first, $e_{2}$, the transition to Frankfort (el/ $\backslash) . e_{4}$ and $e_{5}$ serve as background to $e_{3}, e_{13}$ is the background of $e_{12}(\mathrm{bg} / \bigcup)$. Finally, the continuation chain $e_{7}-e_{8}$ is a flashback seen from the perspective $e_{6}$.

We define the accessibility of an event as reference event in such a way that, having processed the whole text, the only accessible event will be $e_{12}$, whereas, in the case of having processed the text till the integration of $e_{8}$, the accessible events will be $e_{8}, e_{6}$ and $e_{2}$ (with decreasing prominence). In this situation $e_{8}$ is the so called actual reference event. When integrating a new sentence, one will try to expand the node represented by the actual referent first. But, finally, what level has to be chosen and what kind of anchoring has to be stored in the next step is decided by the system rules. So, for instance, in the case of (6), by purely linguistic reasons, $e_{0}$ cannot be related to the actual reference event $e_{8}$, because there is a tense switch from past perfect to simple past between $e_{8}$ and $e_{\theta}$ which indicates the termination of the flashback. This results in the re-initialization of the level the flashback started from as the level providing the new actual referent. In the case of (6) this is $e_{0}$ which is a member of an elaboration. Actual elaborations may be terminated by knowledge indicating that expanding the structure at the actual level is not plausible or even not possible. In this case the system will try to relate the new event with respect to the (accessible) events of higher levels of the elaboration hierarchy. Since, with respect to (6), $e_{9}$ is compatible with $e_{2}$ the system has no reason to terminate the level marked by $e_{\beta}$. We think that the data confirm the concept of a hierarchy of reference events as used in the system. The exact definition of the accessible reference events of a $T$-list resides in the definition of the specific access-functions for $T$-lists that we sketch 
in section $5 .^{2}$

In fact, in the LILOG inplementation, the items of the index, i.e. of the T-list, do not consist of the pure cvents. They are terms with different slots filled by the relevant temporal information such as the tense form which introduces the corresponding event, the aspect, the actual speech time etc. Skipping such technical details, the simplified syntax of the T-list is the following:

$$
\text { T-list }:=\quad\left[\begin{array}{l}
{[\mathrm{E}(\mathrm{D}-\text { list }) \mid \mathrm{T}-\text { List }]} \\
\mathbf{3}
\end{array}\right.
$$

where

with

$\mathrm{E}$ : the discourse referent for the event, and

D-List: the list of the items depending on the event,

where

$$
\begin{aligned}
\text { D-List : }= & {[] ; } \\
& {\left[\mathrm{bg}\left(\left[\mathrm{E}\left([) \mid \mathrm{T}^{\prime}-\text { List }\right]\right) \mid \mathrm{D}-\text { List }\right] ;\right.} \\
& {[\mathrm{fb}([\mathrm{E}(\mathrm{D}-\text { List }) \mid \mathrm{T}-\text { List }]) \mid \mathrm{D} \text {-List }] ; } \\
& {[\mathrm{e}([\mathrm{E}(\mathrm{D}-\text { List }) \mid \mathrm{T}-\text { List }]) \mid \mathrm{D}-\text { List }] ; }
\end{aligned}
$$

Here, of course, embeddings which are given by the dimensions $b y$. $f b$, el in turn stand for backgronnd, flashlback and elaboration. 'T'-List is a T-list where each D-list is the empty list. We use T'-List for the items in bg-lists, because we do not think that a background give rise to further embeddings. The neighborlood of T-list items of the same level signifies continuation, except the case where such a list is fronted by $b g$. In this case, in the corresponding DRS the elements of the list include the same event and, therefore, overlap each other. Continuation is reflected in the reversed order, because we use T-list as a stack. For (6), according to ( 7 ), we write (8). Here and in the following we abbreviate $\mathrm{E}([])$ by $\mathrm{E}$ :

(8) $[\mathrm{e} 12([\mathrm{bg}([\mathrm{e} 13])])$,

e11,

e10, e2 $([\mathrm{el}([\mathrm{e} 9, \mathrm{e} 6([\mathrm{fb}([\mathrm{e} 8, \mathrm{e} 7])]), \mathrm{e} 3([\mathrm{bg}([\mathrm{e} 5, \mathrm{e} 4])])])$, e1]

Of course, there are other discourse relations as those mentioned. Especially, if one tries to analyse the structure of texts on a more fine-grained level than the purely temporal one, the relations described are not sufficient, others like explanation, contrast etc. are needed. ${ }^{3}$ For lack of space, here, we can nothing say about these. The relations described are those

\footnotetext{
${ }^{2}$ Anclioring new events to nom-accessible reference events clearly is possible, provided there is additional information to motivate this choice, such as, for instance, definite descriptions using event nominalizations in order to establish coreferentiality. But, at present, such possibilities are not implemented.

${ }^{3}$ Partly for other purposes discourse or rhetorical relations are used. for instance, in Grosz and Sidner(1985), Thompson and Mann (1987).
}

dealt with in the LILOG system.

\section{Background Knowledge}

The LILOG knowledge base provides us with a sort hierarchy for cliscourse referents which is related with respect to events to the classification given in Vendler(1967). This sort hierarchy is used as one of several information packages within the temporal resolution. With respect to the hierarchy we mainly use calendar knowledge and incompatibilty-statements about event types. For instance, an event introduced with the location time 1987 (or with subintervals of 1987) cannot overlap with an event with location time 1988. Exactly the same is true when the new event has an event type which is incompatible with the type of the reference event. A SWIMMING-event is incompatible with a WRITING-event if the relevant thematic roles are the same, i.e., here, the agent-role. Of course, when formulating such incompatibilities one tries to nake use of the sort hierarchy. One will define the incompatibility for very general sorts (if possible) so that it is inherited by subsorts.

Knowledge of this kind is hard information so to speak. In addition to this, the system uses default knowledge about event types which is similar to what is called event frames (cf. Minsky (1975), Schank and Abelson (1977), Bartsch (1987) and others). Typical (simplified) examples of the two kinds of knowledge are (9) and (10):

(9) $\forall E_{1} \in M O V E, L \in L O C$

$\left(L \subseteq \operatorname{path}\left(E_{1}\right) \rightarrow \exists E_{2} \in P L A C E D-A T\right.$

$\left(\right.$ theme $\left(E_{1}\right)=$ theme $\left.\left.\left(E_{2}\right) \wedge E_{2} \subseteq E_{1}\right)\right)$

(10) $\forall E_{1} \in T U R N, E_{2} \in B E I N G-L I G H T E D$ $\left(\right.$ object $\left(E_{1}\right) \in$ LIGHT-SWITCH $\wedge E_{2}$ meets $E_{1} \rightarrow$ D $\exists E_{3} \in B E I N G-D A R K \quad\left(E_{1}\right.$ meets $\left.\left.E_{3}\right)\right)$

(9) regulates the localization of the theme of a movement. From this rule we infer the existence of different PLACED-AT-states for the theme within the movement. (10) predicts that normally $(D$ for default) turning a switch when it is lighted results in darkness.

\section{Temporal Resolution}

The construction of the semantic representation for a discourse proceeds in several stages. I shall skip here the steps leading to the DRS of a sentence. But it has to be stressed that the result of sentence processing is not a completed DRS. There may be variables which have to be instantiated in the process of integrating the sentence-DRS into the text-DRS.

The sentence-DRS is understood as the input of the aspect-calculus of the prototype which has to compute the aspect of the events of the DRS. Therefore it uses the information provided by the thematic roles. The starting point is the aspect-entry for the 
verb in the lexicon. It can be overwritten by specific role information and morphological information with regard to aspect. For the sake of simplicity in the following we assume that there are just two contrasting aspects corresponding to the main sorts of the upper structure of the event sort hierarchy, the leterogeneous events proper (ev) and the homogeneous states (st). The aspect calculus used is based on ideas from lirifka (1987). ${ }^{4}$ So, for Peter wrote a book we will get the aspect value $e v$, whereas for Peter wrote books we will get st. For the example (6) we get that $e_{4}, e_{5}, e_{1} 3$ are states, the rest will be analyzed as events proper. After the computation of the aspect we come up with an indexed DRS as described in the second and third section with the value of the aspect feature instantiated.

Within the process of anchoring we use several access-functions with respect to the T-list. Among other things these are act-ref(T-list) which gives us the most topicalized item in the list, the actual referent, el-embedder( $T$-list,$E$ ), fb-embedder (T-list, $E$ ) which give us the points elaborations and flashbacks containing $E$ start from. It lias to be stressed that reference events have to be events proper. They cannot be states. The actual referent is the first element of the stack, provided that this element does not contain flaslback- or elaboration-emberldings. If it does we start the recursive call of the search procedure with respect to the first of these embedded lists. tense $(E)$, aspect $(E)$, and $t p(E)$ give us the values of the tense and aspect features ( $s p, p p f, e v, s t$ : simple past, past perfect, event and state) and the temporal perspective from which $E$ is seen.

In order to decide the anchoring of new events, the rules of the system make use of three filters:

(F1) the tense/aspect filter which decides whether a particular discourse relation is possible with respect to tense and aspect,

(F2) the "consistency" filter, which decides whether the choice of a particular discourse relation is logically compatible with the information of the preceding text against the background of the information from the inference component (this consists mainly of testing sort subsumptions in the context of temporal incompatibilities between calendar units and event sorts), and

(F3) the evidence filter which tries to support the relation tested by means of rules like (9) and (10).

To illustrate the system we restrict ourselves to some simple cases (compare figure 1). We suppose that the new sentence introduces just one event and we only sketch simplified versions of the rules for continuation, 1.), for elaboration, 2.), for flashback,

\footnotetext{
TFor a detailed description of the calculus refer to Eberle (1991).
}

3.), for causally introduced background, 4.), for background, 5.), for termination of elaborations, 6.), and for the termination of flashbacks, 7.). We skip rules which deal with specific subcases of these relations. For instance, we skip rules which deal with dummy referents $t$ introduced by the causal background and rules which allow for more precise temporal localizations of the new events. We use rewrite rules which depict the amalgamation of a sentence DRS with a text DRS.

In rule 1.) of figure $1,\left(^{*}\right)$ reflects the particular case where $e_{r e f}$ is member of an elaboration. If it is not, the corresponding condition in $K_{n+1}$ is omitted.

In the cases 1.) - 3.) (F1) requires that $e_{S}$ is heterogeneous $\left(\operatorname{aspect}\left(e_{S}\right)=e v\right)$. The tense forms of reference- and new event have to be identical (tense $\left.\left(e_{S}\right)=\operatorname{tense}\left(e_{\text {ref }}\right)\right)$. In addition, $(\mathrm{F} 1)$ accepts 3.), if tense $\left(e_{r e f}\right)=s p$ and tense $\left.\left(e_{S}\right)=p p f\right)$. This arrangement allows also for continuation and elaboration on flasliback levels and for iteration of flashbacks. For 4.) and 5.) the tense forms must be identical. In contrast to 1.) - 3.), it is required that aspect $\left(e_{S}\right)=s t$.

(F2) should be clear in all cases. For acceptance, the tested solutions have to be consistent.

With respect to the cases 1.) - 4.), (F3) accepts the relation tested, if some evidence for this relation can be inferred from the representation of the preceding text on the basis of the background knowledge. In addition, in the cases 1.) - 3.) (F3) also accepts the relation, if evidence for the competing relations cannot be entailed. In the case of tense $\left(e_{S}\right)=$ tense $\left(c_{\text {ref }}\right)=p p f$, flashback, elaboration and continuation are pairwise competing relations. In the case of tense $\left(e_{S}\right)=\operatorname{tense}\left(e_{, e f}\right)=s p$, flashback is ruled out as competing relation with respect to elaboration and continuation. In the case of 5.), background, and 3.) with tense $\left(e_{S}\right) \neq$ tense $\left(e_{r e f}\right)$ (F3) is empty.

Evidence for a particular relation $X$ can be inferred, if there exists an event sort $P$ which is more general than the event description from $D R S_{S}$ with respect to the event sort hierarchy, such that the knowledge base (KB) together with the representation of the preceding text $\left(D R S_{n}\right)$ predicts an event $e$ auf type $P$ temporally related to $e_{\text {ref }}$ in a way significant for $X$, and that $\mathrm{KB}, D R S_{n}$ do not entail the corresponding statement with respect to the competing relations of $X$. Formally, for instance with respect to $X=$ "continuation", $X$ is evident, if for $P$ with $\forall e\left(D R S_{S}\left[e_{S} / e\right] \rightarrow P(e)\right)$, we can infer that $\exists e\left(P(e) \wedge e_{\text {ref }} \prec e\right)$, but neither that $\exists e \quad\left(P(e) \wedge e \subseteq e_{\text {ref }}\right)$ nor $\exists e \quad\left(P(e) \wedge e \prec e_{\text {ref }}\right)$.

The whole system is implemented in Prolog. So the order of the rules is important. Thus, we get a preference relation over the allowed readings. Continuation is preferred to elaboration which is preferred to flashback (in the case that all alternatives seem possible and that they are stipported by (F3)). With respect to 6.) and 7.), we see that local level expansion is preferred to the anchoring of the new event at 
$K_{n+1}=K_{n} \cup K_{S}$

$\cup\left[\begin{array}{l}e_{\text {ref }} \prec e_{s} \\ e_{s} \prec e_{t p} \\ e_{s} \subseteq e_{e l r e f}(*)\end{array}\right]$

$T_{n+1}=\ldots\left[\ldots e_{s}, e_{\text {ref } f}, \ldots\right] \ldots$

2. Elaboration:

$U_{n+1}=U_{n} \cup U_{S}$

$K_{n+1}=K_{n} \cup K_{S} \cup e_{S} \subseteq e_{\text {ref }}$

(F1) (F2) (F3)

$\operatorname{akt-ref}\left(T_{n}\right)=e_{\text {ref }}$

$T_{S}=\left[e_{S}\right]$

$T_{n+1}=$.[.ee $\left.e_{\text {ref }}\left(\left[e l\left(\left[e_{S}\right]\right),.\right]\right),.\right]$.

3. Flasliback:

$U_{n+1}=U_{n} \cup U_{S}$

$K_{n+1}=K_{n} \cup I_{S} \cup e_{S} \prec e_{r e f}$

$T_{n+1}=$.[.e.ef $\left.\left(\left[f b\left(\left[e_{S}\right]\right),.\right]\right),.\right]$.

(F1) (F2) (F3) akt-ref $\left(T_{n 1}\right)=e_{\text {,ef }}$

$T_{S}=\left\lfloor e_{S}\right\rceil$

4. Causally introduced Background:

$U_{n+1}=U_{n} \cup U_{S}$

$\Lambda_{n+1}=\Lambda_{n} \cup \Lambda_{s}$

$U \begin{aligned} & e_{\text {ref }} \prec t \\ & e_{\text {ref }} \prec e_{S} \\ & t \subseteq e_{S}\end{aligned}$

(F1) (F2) (F3)

$a k t-\operatorname{ref}\left(T_{n}\right)=e_{\operatorname{ref}}$

$T_{S}=\left[e_{S}\right]$

$T_{n+1}=.\left[t\left(\left[b g([e s]), c_{n e f},.\right]\right),.\right]$.

5. Background:
$U_{n+1}=U_{n} \cup U_{S}$
$\Pi_{n+1}=K_{n} \cup K_{S} \cup\left[e_{r e f} \subseteq e_{s}\right.$
$T_{n+1}=.\left(e_{\text {ref }}\left(\left[\operatorname{bg}\left(\left[e_{S}\right]\right), .\right]\right)_{4}.\right]$

$6 . / 7$. Return from elaborations/flashbacks:

restart the system

with eref'

instead of $e_{r o f}$

akt $-\operatorname{ref}\left(T_{n}\right)=e_{\text {ref }}$

$T_{S}=\left\lfloor e_{S}\right\rfloor$

akt-ref $\left(T_{n}\right)=e_{\text {ref }}$

6.) el-embedler $\left(T_{n}, e_{\text {ref }}\right)=\varepsilon_{\text {ref }} \quad T_{S}=\left\lfloor e_{S} \mid\right.$

7.) $f b$-rembedder $\left(T_{n}, e_{\text {ref }}\right)=e_{\text {ref }}$

Figure 1: resolution rules

higher levels of the hierarchy. This strategy seems to be validated by the text phenomena.

For illustration, we briefly sketch the effects of the rules when applied to the sample text (6). Leaving Paris- and Going to Frankfort-events camot overlap. Therefore, (F2) rules out the alteruative "elaboration" for $e_{2}$ with respect to $e_{1}$ and the remaining competing "continuation" is chosen. Further support by (F3) is not needed in this case. For travels $t$ to foreign conntries we can infer the existence of $P L A C E D$ $A T$ 'states $c^{\prime}$ for the agent or thenc of the travelling' event and for the borders contained in the path of the travelling such that $e^{\prime} \subseteq e$ (compare the KB rtile (9)). Since the description of $e_{3}$, the stop at the border, is subsumed by the more general PLACED-ATsort, (F3) - in the absence of corresponding evidences for the competing relation of continuation - supports "elaboration". Since for the states $e_{4}, e_{5}, e_{13}$ the alterliative of "causally introduced background" is not evident, they are anchored by means of the rule 5.). In the case of $e_{0}$ and $e_{0}$, the fact that evidence for "elaboration" is missing selects for "continuation". e.10 which is situated in Frankfort cannot be part of the travel. For this reason anchoring $e_{10}$ with respect to the level of $c_{r e f}$, which actually is $e_{\theta}$, is not possible. Therefore, with rule 6.), we have to climb up the elaboration hierarchy. The anchoring of $e_{7}, e_{8}, e_{11}$ and $e_{12}$ should be clear on the basis of what we have said so far.

In order to arrive at alternative readings of a text we use a specific backtracking rontine which can suppress the inpact of (F3) for the resolution.

\section{Conclusion}

The described system computes the event structure of a discourse. Since we think that the semantics of tense and aspect is not sufficient to establish the temporal disconrse meaning, we use backgronnd knowledge to disambiguate between different readings.

The system is incomplete at present. The fragnent it deals with doesn't allow for relative clauses. In addition the interaction between NP-resolution and temporal resolution is not available. Some temporally relevint discourse relations are not treated. The logic underlying the preference relation is only rudimen. tary sketched. The approach to temporal resolution 
presented here is a condensed version of that suggested in Eberle (1991). The corresponding implementation for the LILOG prototype dates back to 1989. Meanwhile, independent of this approach, similar suggestions have been made (cf. Asher and Lascarides (1991)). In the work of Asher and Lascarides the underlying theory of non-monotonic reasoning is MASH (cf. Asher and Morreau (1991)). It is projected to extract a suited proof theory from MASH for the purpose of refining the temporal resolution component suggested. ${ }^{3}$

\section{References}

[1] Asher, N., Lascarides. A. (1991): Discourse Relations and Defeasible Knowledge. In: Proceedings of ACL,1991

[2] Asher, N., Morreau. M. (1991): Common Sense Entailment: A Modal Theory of Non-monotonic Reasoning. In: Proceedings of IJCAIL, Sydney 1991

[3] Bartsch, R.(1987): Frame Representations and Discourse Representations. ITLI Prepublication Series 87-02. University of Amsterdam.

[4] Dowty, D.(1986): The Effects of Aspectual Class on the Temporal Structure of Discourse: Semantics or Pragmatics?. In: Linguistics and Philosoplyy Vol.9,No.1 (1986) pp.37-62

[5] Eberle, K. (1991): Ereignisse: Thre Logik und Ontologie aus textsemantischer Sicht. Dissertation Universität Stuttgart, 1991

[6] Eberle, K., Kasper, W.(1989): Tenses as Anaphort. In: Proceedings of E-ACL, Manchester 1989

[7] Grosz, B., Sidner, C.(1985): The Structure of Discourse Structure SRI-technical note 369, 1985

[8] Hinriclss, E.(1986): Temporal Anaphora in Discoutses of English. In: Linguistics and Philosoplyy Vol.9,No.1 (1986) pp.63-82

[9] Kamp, H.(1981): A Theory of Truth and Semantic Representation. In: Groenendeijk et al (eds.) Formal Methods in the Study of Language. Mathematical Centre Tract, Amsterdam

[10] Kamp, H., Rohrer, C. (1983): Tense in Texts. In: Bäıerle, R.,Schwarze, C.,von Stechow, A. (eds.), Meaning, Use and Interpretation of Language, Berlin, de Gruyter

[11] Kamp. H., Rolirer, C. (1985): Temporal Reference in French. Ms. Stuttgart

\footnotetext{
since $\mathrm{M}$. Morreau is member of our institute and N. Asher visiting professor in Stuttgart, this can be done in close connection witl the authors of MASH.
}

(12) Krifka, M. (1987): Nominalreferenz und Zeitkonstitution. Zur Semantik von Massentermen, Pluraltermen und Aspektklassen. Dissertation Universität München, 1987

[13] Minsky, M.(1975): A Framework for Representing Knowledge. In: Winston,P. (ed.) The Psychology of Computer Vision. McGraw-Hill, New York.

[14] Moens, M., Steedman, M. (1986): The Temporal Dimension in Information Modelling and Natural Language Processing, Acord Deliverable 2.5, Edinburgh, 1986

[15] Moens, M., Steedman, M. (1988): Temporal On. tology and Temporal Reference, in: Computational Linguistics 14(2), 1988

[16] Partee, B. (1984): Nominal and Temporal Anaphora, in: Linguistics and Philosophy Vol.7,No.3 (1984) pp.243-287

[17] Reichenbach, H. (1947): Elements of Symbolic Logic. New York, 1947

[18] Reyle, U. (1986): Zeit und Aspekt bei der Verarbeitung natïrlicher Symachen. Dissertation Institut für Linguistik der Universität Stuttgart,1986

[19] Schank, R., Abelson, R. (1977): Scripts, Plans, Goals and Understanding. Hillsdale, N.J. LEA.

[20] Thompson, S.,Mann, W.C. (1987): Rhetorical Structure Theory: A Framework for the Analysis of Texts. In: International Pragmatics Association Papers in Pragmatics, Vol.1, pp.79-105, 1987

[21] Vendler, Z. (1967): Linguistics in Philosophy, Cornell University Press, Ithaca, New York

[22] Webber, B.L. (1988): Tense as Discourse Anaphor, in: Computational Linguistics 14(2), 1988 